Classification

Physics Abstracts

$07.60 \mathrm{j}-42.80 \mathrm{f}-78.20^{-} \mathrm{e}-81.70^{+} \mathrm{r}-85.30 \mathrm{z}-86.50 \mathrm{j}$

\title{
Microscopies de champ proche optique : application aux semiconducteurs
}

\author{
Jean Pierre Fillard, Michel Castagné et Christel Prioleau \\ Laboratoire LINCS - CEM, Université Montpellier 2, 34095 Montpellier Cedex 05, France
}

(Reçu le 4 juillet; accepté le 26 octobre 1994)

\begin{abstract}
Résumé. - Les microscopies optiques de champ proche (PSTM ou SNOM) sont maintenant bien connues même si leur mise en oeuvre, leurs performances, l'interprétation des images sont encore sujets à discussion. Cette communication est destinée à décrire le cas particulier des semiconducteurs qui, du fait de leur indice optique élevé, présentent des propriétés et des problèmes spécifiques. On montrera que l'utilisation de pointes semiconductrices transparentes de type AFM peut être favorablement envisagée pour des mesures optiques et que les performances espérées en résolution se trouvent trés largement améliorées. On présentera les premières investigations comparatives de transmission optique faites sur des pointes en Nitrure de Silicium et en Silicium dopé, à partir d'une onde évanescente, sur une surface plane de semiconducteur. On montrera en particulier que l'ouverture relative très faible des pointes en Silicium introduit un effet que nous interprétons comme la première illustration d'un mécanisme tunnel photonique résonnant.
\end{abstract}

\begin{abstract}
Near field optical microscopy has become largely investigated even if the implementation, the specifications, the interpretation of images are not satisfactorily mastered. This paper describes the particular situation of semiconductors which behaves differently because of their high refractive index. Transparent semiconductor AFM tips can be used as optical transducers of very high performances. First investigations on comparative transmission properties are presented corresponding to silicon nitride tips and also doped silicon tips on a InP surface. It is shown that the exceptionnally small relative aperture on the Si tips induces a new behaviour which we interpret as the first evidence of a photonic resonnant tunneling effect.
\end{abstract}

\section{Introduction.}

Les semiconducteurs traditionnels comme le Silicium ou l'Arséniure de Gallium ont des indices optiques élevés $(3<n<4)$, ils peuvent être conducteurs ou résistifs mais sont en général transparents dans le proche infra rouge $(1-1.5 \mu \mathrm{m})$.

On a montré que les ondes évanescentes réalisables par "Frustrated Total Internal Reflection" (TIR) sur semiconducteur [1] ont des longueurs critiques de décroissance $d_{c}$ qui peuvent être très 
courtes ; bien que la relation directe avec la "résolution" ne puisse être en général clairement exprimée, $d_{c}$ reste quand même une indication de la qualité des images.

Les semiconducteurs, d'autre part, sont les matériaux les mieux maitrisés pour la réalisation d'objets nanométriques (micromachining [2] sur Silicium). Il est donc naturel de rechercher une sonde qui soit un petit objet en matériau semiconducteur : c'est le cas des pointes AFM disponibles sur le marché. On a démontré (NF Van Hulst [3], D. Courjon [4], J.P. Fillard [5]) que les pointes SiN sont capables de capter l'onde évanescente [6] jusque dans le domaine infra-rouge. On peut donc extrapoler une utilisation optique in situ dans le cadre d'un "capteur intelligent". Nos travaux montrent qu'on peut aller plus loin, jusqu'à la notion de "nanorobot" photonique [7] : le “piezolever" récemment proposé [8] par PSI n'est qu'un premier pas dans la direction d'une intégration opto-électronique plus importante. Cette communication présente les résultats récents obtenus à Montpellier sur les propriétés optiques des pointes AFM utilisées en infrarouge sur InP. On montrera les différences essentielles observées expérimentalement entre les pointes $\mathrm{SiN}$ et les pointes $\mathrm{Si}$.

\section{Les pointes AFM.}

Dans la technologie d'élaboration des pointes pour la microscopie AFM on distingue schématiquement deux catégories distinctes :

2.1 LES POINTES EN NITRURE DE SILICIUM. - Elles sont obtenues (Fig. 1) par des techniques de dépôt de Nitrure de Silicium (low stress silicon rich LPCVD ou PECVD) à partir de "moules" creusés dans un substrat massif de silicium (100). Les plans d'attaque préférentielle (111) du silicium donnent des pyramides creuses à 4 faces, d'axe $<100>$ avec des angles directeurs de $37,5^{\circ}$. Le nitrure déposé en film mince est ensuite dégagé par élimination du Silicium ; c'est un matériau transparent de l'U.V au visible. Ces pointes évidemment sont creuses et l'épaisseur des parois est inférieure au micron.

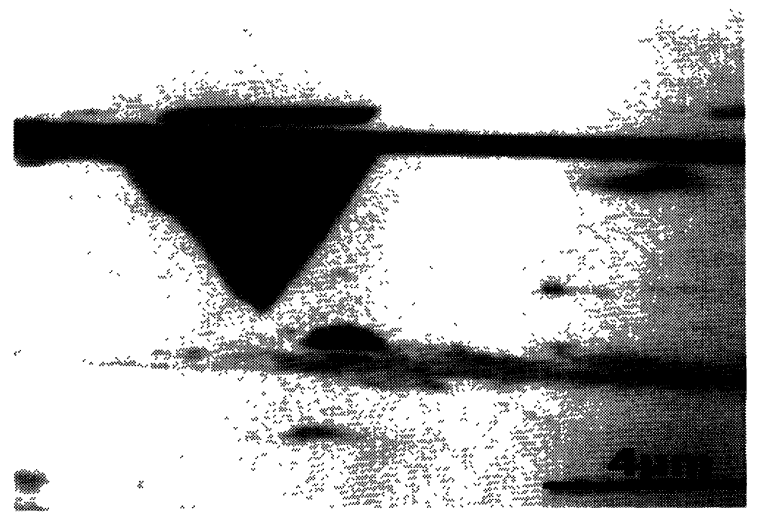

Fig. 1. - Pointes pyramidales en Nitrure de Silicium (document PSI).

Les rayons de courbure au sommet sont relativement larges $(a \simeq 50 \mathrm{~nm})$ et le rapport d'aspect de la pointe est de l'ordre de 1:1. Les pointes sont portées par un support en pyrex et leur résistivité 
électrique est élevée. En général ces pointes sont dorées sur la face arrière. Ce film mince métallique affecte peu la transmission [5] mais peut éventuellement être éliminé facilement.

2.2 LES POINTES EN SILICIUM. - Elles sont généralement obtenues [9] à partir de Si dopé ( $n \simeq$ $10^{20} \mathrm{~cm}^{-3}$ ) au Bore. Leur profil (Figs. 2a et $2 \mathrm{~b}$ ) résulte d'une opération de photolithographie et lift-off par attaque chimique humide et/ou par plasma réactif. Lutilisation de réations anisotropes permet aussi d'utiliser sélectivement les plans cristallins, par example sur un wafer orienté < $100>$ on peut faire ressortir une pointe d'axe $<100>$ avec des facettes d'indices élévés. La combinaison des méthodes permet d'ajuster la forme générale de façon assez variée pour obtenir soit des pointes à grand rapport d'aspect (3:1) soit de terminer la pyramide par une pointe "aiguisée" d'angle trés faible.
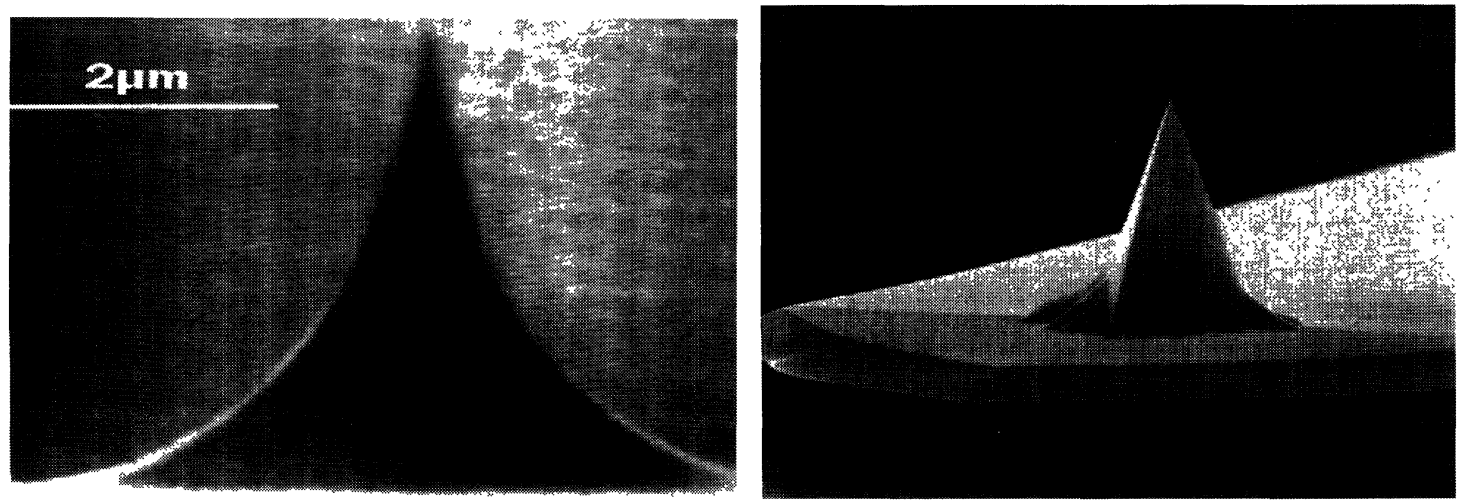

Fig. 2. - a) Pointe conique (document PSI), b) Pointe "sharpened" (communication Nanosensor).

Les pointes en Silicium sont évidemment massives, les rayons de courbure sont inférieurs à 20 $\mathrm{nm}$ et les angles au sommet inférieurs à $20^{\circ}$.

Le domaine de transparence du Silicium est assurément établi pour les transitions d'énergies inférieures à la bande interdite $(\lambda>1,1 \mu \mathrm{m})$ mais la faible épaisseur de matériau à traverser et la variation lente en énergie des transitions indirectes du Silicium font que les pointes transmettent encore suffisamment jusqu'à $\lambda=1,05 \mu \mathrm{m}$. Cette propriété nous a permis d'utiliser indifféremment deux lasers YAG-Nd à $1,06 \mu \mathrm{m}$ et à $1,32 \mu \mathrm{m}$.

Nous avons utilisé dans ce travail des pointes de fabrications diverses dont les leviers avaient des coëfficients de force très différents (de 0,02 à $22 \mathrm{~N} / \mathrm{m}$ ).

\section{Capture par pointes SiN.}

Ces pointes ont été utilisées $[3,4,6]$ dans le visible, sur des surfaces de verre, pour des angles d'incidence larges $\left(45-65^{\circ}\right)$; les fonctions de proximité optiques suivent de façon satisfaisante le modèle classique [6] de la capture "diélectrique" de l'onde évanescente.

Cette observation a été aussi retrouvée avec des surfaces semiconductrices d'InP- Fe semiisolant [5]. Sur la figure 3 on voit l'organisation générale de cette expérimen- tation en proche infra rouge $(\lambda=1.06 \mu \mathrm{m}$ et $\lambda=1.32 \mu \mathrm{m})$. 


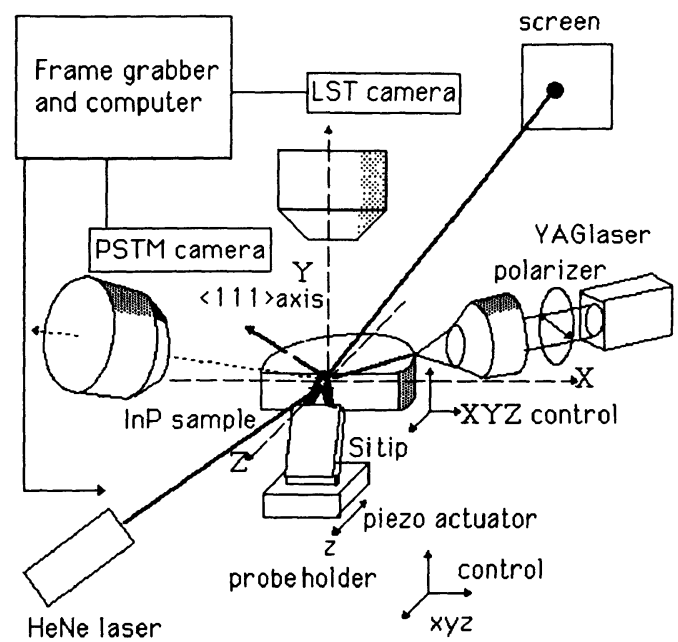

Fig. 3. - Schéma de l'organisation expérimentale de PSTM.

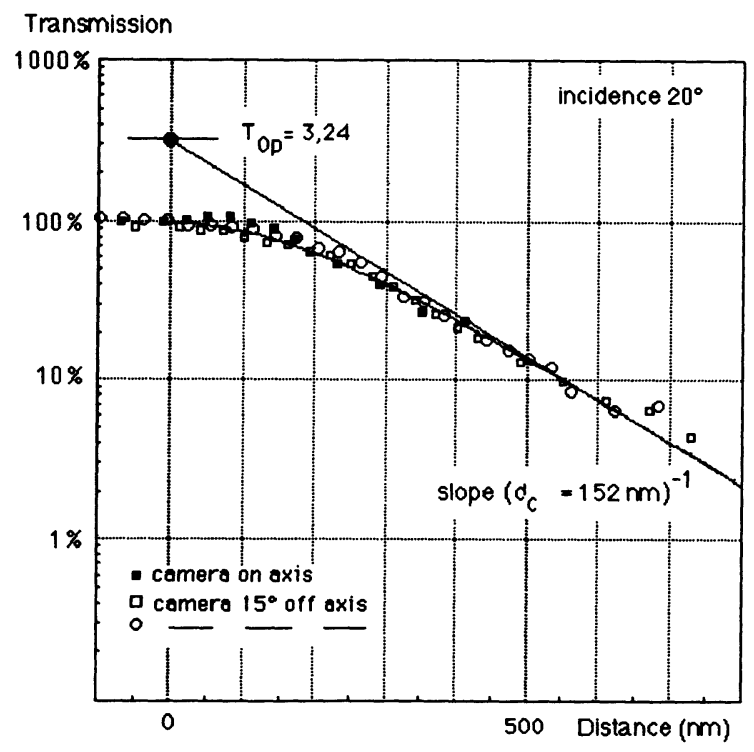

Fig. 4. - Fonction de proximité mesurée avec une pointe SiN sur une surface d'InP avec une incidence de $20^{\circ}$ et à $\lambda=1.06 \mu \mathrm{m}$.

Les indices élevés de la pointe et du substrat permettent ici de travailler avec des angles d'incidence beaucoup plus faibles $\left(20^{\circ}\right)$; pour un $d_{\mathrm{c}}$ comparable, on dispose donc d'une "réserve de résolution" importante en allant vers de plus grandes incidences.

Les résultats sont représentés sur la figure 4, ils recoupent bien le modèle théorique de capture par une structure stratifiée, établi à partir des equations de Maxwell et les conditions aux limites. 


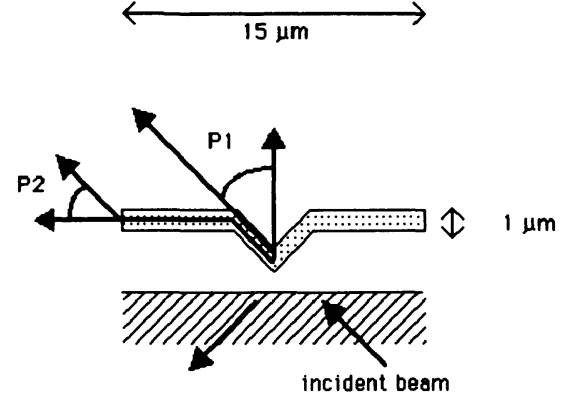

cantilever front section

Fig. 5. - Capture de l'onde évanescente par une pointe SiN.

Par contre on a montré que cette capture se fait sélectivement dans chacun des murs de cette pyramide (Fig. 5) avec un guidage du photon dans la direction du moment du photon incident. Aucune flexion du levier n'a été observée avant d'arriver au contact avec la surface, pourvu que celle-ci soit électrostatiquement neutre ; en général la pellicule d'humidité résiduelle suffit à assurer cet équilibre au bout de quelques minutes.

\section{Capture par pointe Silicium.}

Une étude semblable a été menée $[10,11]$ avec des pointes Silicium.

Les résultats obtenus sont radicalement différents : le comportement pseudo exponentiel disparait au profit d'une décroissance plus lente avec des ondulations assez régulières en $\lambda / 2$ observables sur la courbe directe (Fig. 6) ou sur le spectre de la transformée de Fourier (Fig. 7).

Une explication peut être trouvée dans l'effet tunnel photonique résonant proposé par Leviatan [12] et repris sous la forme de "photons virtuels" par Hori [13].

Louverture standard $a / \lambda$ de nos pointes est ici exceptionnellement petite $(<0,01)$ par rapport à toutes les expériences précédentes, d'une part parce que $a$ est petit $(10 \mathrm{~nm})$ mais aussi parce que $\lambda$ est grand $(1,06$ ou $1,32 \mu \mathrm{m})$.

La résonance sur une ouverture de si petite taille introduit des pics très intenses de transmission [12]. Nous serions donc ici dans la situation d'un phénomène nouveau de transfert tunnel photonique résonnant.

Lexistence de ces pics (ou de ces marches) ne favorise pas la décroissance rapide de la courbe avec la distance ; ceci semble donc indiquer que l'utilisation des pointes Si pour l'imagerie PSTM de haute résolution ne sera pas facilitée. Davantage d'investigations expérimentales sont toutefois indispensables avant de pousser plus loin les conclusions ; on peut cependant remarquer de façon générale à propos de cette étude que la réduction de taille de la pointe n'aboutit pas automatiquement à une amélioration de la résolution.

\section{Conclusion.}

Ces premiers résultats montrent bien que les pointes AFM sont des véhicules photoniques très intéressants pour les nanotechnologies des semiconducteurs. Les applications prévisibles sont 

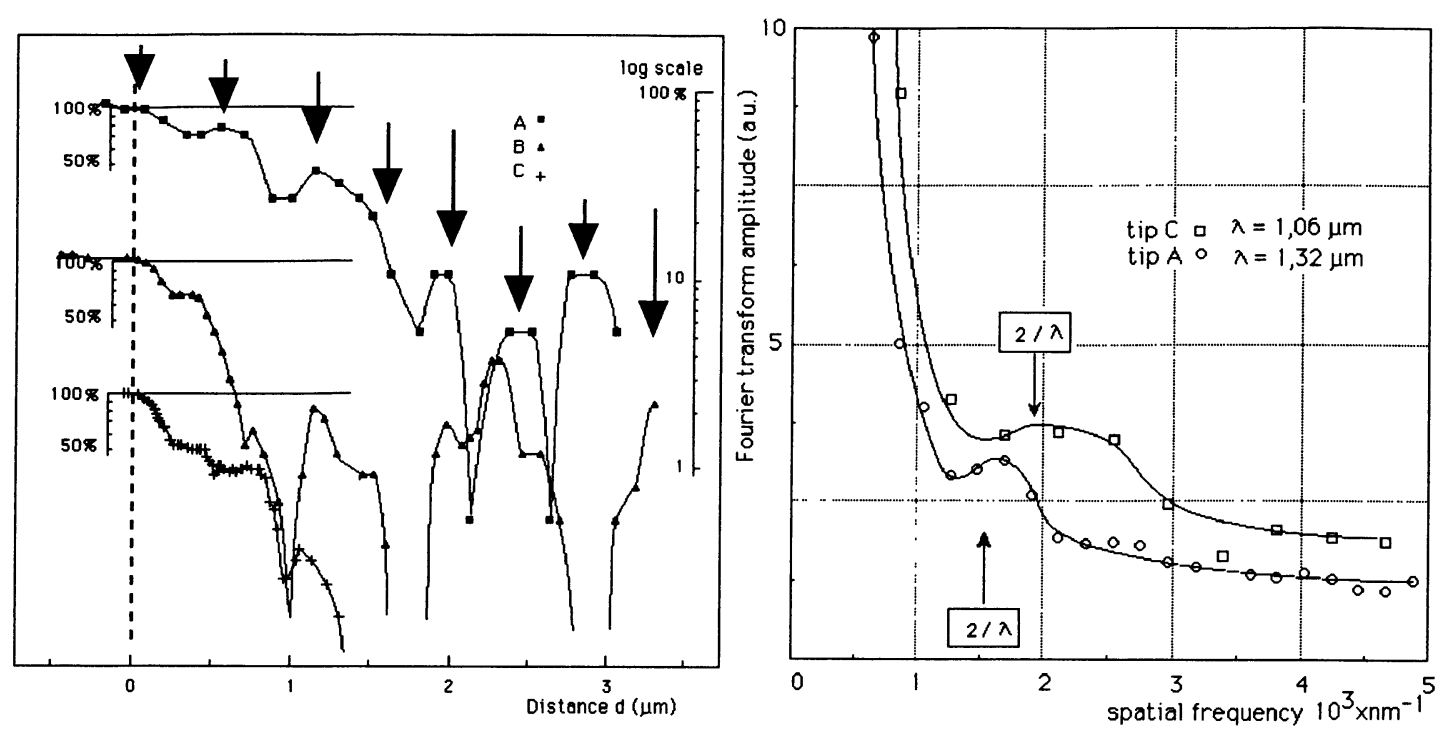

Fig. 6. - Fonction de proximité de pointes Si sur une surface d'InP montrant l'effet tunnel résonnant.

Fig. 7. - Transformées de Fourier de fonctions de proximité à 1, $06 \mu \mathrm{m}$ et $1,32 \mu \mathrm{m}$.

nombreuses tant vers l'intervention photonique localisée que vers les couplages optoélectroniques à très haute résolution.

Ces techniques photoniques peuvent aussi se combiner avec des effets électriques collectifs [14] ou individualisés dans des structures quantiques de confinement.

\section{Remerciements.}

Ce travail a été réalisé dans le cadre de l'action programme CNET "Traitement du signal optique" et du contrat 9135169 Les auteurs remercient le Dr. O. Wolter (Nanosensor Co) pour sa collaboration.

\section{Bibliographie}

[1] Fillard J.P., Prioleau C., Lussert J.M., Castagné M., Bonnafé J., "PSTM images for semiconductor technologies", in Defect Recognition and Image Processing, J. Jimenez, Vol. 135 of Inst. Phys. Conf. Series (IOP London 1994) 247-251.

[2] Bryzek J., Petersen K., McCulley W., "micromachines on the march", IEEE Spectrum (20 mai, 1994).

[3] Van Hulst N.F., Moers M.H.P., Noordman O.F.J., Tack R.G., Segerink F.B., Bölger B., "Near field optical microscopy using a Silicon Nitride probe", Appl. Phys. Lett. 62 (1993) 461.

[4] Baïda F., Courjon D., Tribillon G., "Combination of a fiber and a Silicon Nitride tip as a bifunctional detector; first results and perspectives", in Near-Field Optics, D.W. Pohl, D. Courjon, Vol. E 242 of NATO Series (Kluwer Acad. Pub. Ed. 1992) 71-78.

[5] Castagné M., Prioleau C., Baudry E., Fillard J.P., Bonnafé J., "Photon tunneling from InP material surface”, 6th Int. Conf. InP and rel. Mat. (Santa Barbara, 1994) Comptes rendus à paraitre. 
[6] Castagné M., Prioleau C., Fillard J.P., "An experimental study of the optical properties of SiN AFM tips in Scanning Tunneling Microscopy", Appl. Opt. à paraitre (1994).

[7] Fillard J.P., Castagné M., “An intelligent optical scanner”, European patent application EP 933057861.

[8] Tortonese M., Barrett R.C., Quate C.A., "Atomic resolution with an atomic force microscope using piezoresistive detection", communication privée à paraitre.

[9] Wolter O., "Micromachined Silicon sensors for scanning force microscopy”, J. Vac. Sc. Tech. B9 (1991) 1353.

[10] Fillard J.P., Castagné M., Prioleau C., Baudry E., Gall P., Bonnafé J., "Photon tunneling scanning microscopy and atomic force microscopy on semiconductor surfaces", 2nd Int. Workshop EXMATEC'94, Parma (1994) Comptes rendus à paraitre.

[11] Fillard J.P., Castagné M., Prioleau C., "AFM Silicon tips as photon tunneling sensors: a resonant evanescent coupling experiment", Appl. Opt. à paraitre (1994).

[12] Leviatan Y., "Electromagnetic coupling between two half space regions separated by two slots perforated in parallel conducting screens", IEEE trans. Microwave Theo. Tech. 36 (1988) 1.

[13] Hori H., "Quantum optical picture of photon STM and proposal of single atom manipulation", Near field optics, D.W. Pohl and D. Courjon Eds., NATO Ser. E 242 (1993) 105.

[14] Mertz J., Hipp M., Mlynek J., Marti O., "Measurement of force induced by an evanescent wave on a semiconductor tip", 2nd Int. Conf. on NFO 2 Raleigh (1993) Comptes rendus à paraitre. 